\title{
Self-duality and Integrable Systems
}

\author{
By \\ Yousuke OHYAMA*
}

\section{$\S 0 . \quad$ Introduction}

In his lectures (1984-85) at Kyoto University, Professor M. Sato presented a program for generalizing the soliton theory ([9]; cf. [10]). The KadomtsevPetviashvili (KP) equation is a typical example of the soliton theory. The KP equation is written in the form of deformation equations of a linear ordinary differential equation. The time evolutions of a solution are interpreted as dynamical motions on an infinite dimensional Grassmann manifold ([7], [9]). The Lie algebra of microdifferential operators of one variable acts on this manifold transitively. He conjectured that any integrable systems can be written in the form of deformation equations of a linear system, and proposed to investigate a deformation of differential equations in higher dimensions. $\mathrm{He}$ showed a simple example of a deformation of holonomic systems in higher dimensions ([9]), and its generalization is treated in [4]. In this paper we study a deformation of $\mathscr{D}$-modules in higher dimensions.

First we review the KP equation. We denote by $\mathcal{E}$ the ring of microdifferential operators of one variable $x$. We fix a microdifferential operator $P$, and denote by $t_{P}$ a time variable with respect to $P$. We study the following evolution equation associated to $P$ :

$$
\frac{\partial W}{\partial t_{P}}+W P=\left(W P W^{-1}\right)_{+} W
$$

where $W=W\left(x, D_{x}\right)=1+\Sigma_{\jmath<0} w_{\jmath}(x) D_{x}^{j} \in \mathcal{E}$. We denote by $\mathscr{W}$ the set of such operators $W$. This space $\mathscr{W}$ is a group by the composition of $\mathcal{E}$. We get the KP-hierarchy taking $P=D_{x}^{n}(u=1,2,3, \cdots)$ in (0.1). The equation (0.1) defines a dynamical motion on $\mathscr{W}$. This infinitesimal action of the Lie algebra $\mathcal{E}$ on $\mathscr{W}$ is transitive.

The purpose of this article is to give a foundation for higher dimensional generalization of the KP hierarchy. Let now $\mathcal{E}$ be the ring of microdifferential operators in several variables. Similarly to the one dimensional case, fixing an operator $P \in \mathcal{E}$, we shall study the following equation

Communicated by M. Kashiwara, January 8, 1990.

* Department of Mathematics, Faculty of Science, Osaka University, Toyonaka, Osaka 560, Japan. 


$$
\frac{\partial W}{\partial t_{P}}+W P=\left(W P W^{-1}\right)_{+} W,
$$

where the operator $W$ is a 0 -th order microdifferential operator. Here we choose a decomposition $\mathcal{E}=\mathscr{D} \oplus \mathcal{E}_{\phi}$ and $\left(W^{T} P W^{-1}\right)_{+} \in \mathscr{D}$ is the component of $W P W^{-1}$ according to this decomposition. In general the equation (0.2) imposes some constraints on the initial value $W\left(t_{P}=0\right)$, since the vector field defined by $(0.2)$ is not tangent to the space $\mathcal{E}(0)$. There is no operator $W_{0}$ such that the equation $(0.2)$ has a solution $W(t) \in \mathcal{E}$ with the initial value $W_{0}$ for any $P \in \mathcal{E}$. We take generators $P$ in (0.2) only in the Lie subalgebra $V$ of $\mathcal{E}$

$$
\begin{aligned}
V=\left\{F_{0}\left(x^{\prime}, D_{0}\right) x_{0}+\right. & \sum_{0 \leqq j<r} F_{j}\left(x^{\prime}, D_{0}\right) D_{j}+E\left(x^{\prime}, D_{0}\right) ; \\
& \left.F_{k}\left(x^{\prime}, D_{0}\right), E\left(x^{\prime}, D_{0}\right) \in \mathcal{E} \text { for } 0 \leqq k<r\right\},
\end{aligned}
$$

where $x^{\prime}=\left(x_{1}, x_{2}, \cdots, x_{r-1}\right)$. This Lie algebra contains the transformation groups both of the self-dual Yang-Mills equations and of the self-dual Einstein equations (see [7], [8]). In $\S 2$ we will determine the subspace $\mathscr{W}$ of $\mathcal{E}(0)$ so that the vector field defined by $(0.2)$ for any $P \in V$ is tangent to $\mathscr{W}$. The space $\mathscr{W}$ is a subgroup in $\mathcal{E}$. The Lie algebra $V$ acts on $\mathscr{W}$ transitively.

In the case of $r=3$, our integrable system is nothing but a composed system of the self-dual Yang-Mills equations and the equations of self-dual metrics on Riemannian manifolds of dimension four. The Lie algebra $V$ acts transitively on the space of self-dual connections on self-dual spaces. Thus we obtain a group-theoretical description of the twistor theory ([1], [5]).

Notations. We use the following notations: $\boldsymbol{Z}$ denotes the set of integers. $\boldsymbol{N}$ denotes the set of non-negative integers. We denote by $\boldsymbol{C}$ the complex number field. We denote by $1_{n}$ the unit matrix of size $n \times n$.

\section{$\S 1$. Deformation $\mathscr{D}$-Modules}

Throughout this paper we shall work in the category of formal power series, $\mathcal{O}=\boldsymbol{C}[[x]]=\boldsymbol{C}\left[\left[x_{0}, x_{1}, \cdots, x_{r-1}\right]\right](r \geqq 2)$. Let $\mathscr{D}$ be the ring of differential operators with coefficients in $\mathcal{O}$. Then every differential operator $P$ of order $m$ can be written as:

$$
P=\sum_{\alpha \in N} a_{r,|\alpha| \leqq m} a_{\alpha}(x) D_{x}^{\alpha},
$$

where $a_{\alpha}(x)$ are elements of $\mathcal{O}, \alpha=\left(\alpha_{0}, \alpha_{1}, \cdots, \alpha_{r-1}\right) \in N^{r},|\alpha|=\alpha_{0}+\alpha_{1}+\cdots+\alpha_{r-1}$, $D_{x}^{\alpha}=D_{0}^{\alpha} D_{1}^{\alpha_{1}} \ldots D_{r-1}^{\alpha_{1}^{-1}}$ and $D_{j}=\partial / \partial x_{j}(j=0,1, \cdots, r-1)$.

The ring $\mathcal{E}$ of formal microdifferential operators is a set of formal Laurent series in $D_{0}, D_{1}, \cdots, D_{r-1}$ with only non-negative powers of $D_{1}, \cdots, D_{r-1}$. The precise definition is as follows. We denote by $\mathcal{E}(m)$ the space of formal series: 


$$
P=\sum_{\alpha \in Z \times \boldsymbol{N}} \sum_{r-1,|\alpha| \leqq m} a_{\alpha}(x) D_{x}^{\alpha}
$$

where $a_{\alpha}$ 's are elements of $\mathcal{O}$, and the summation is taken through $\alpha=\left(\alpha_{0}, \alpha_{1}\right.$, $\left.\cdots, \alpha_{r-1}\right)$, where $\alpha_{0} \in \boldsymbol{Z}, \alpha_{1} \in \boldsymbol{N}, \cdots, \alpha_{r-1} \in \boldsymbol{N}$. We set

$$
\mathcal{E}=\bigcup_{m \in \boldsymbol{Z}} \mathcal{E}(m) \text {. }
$$

We endow the $\mathcal{O}$-module $\mathcal{E}$ with a structure of ring by extending the Leibniz formula. For two elements $P=\Sigma_{\alpha} a_{\alpha} D_{x}^{\alpha}$ and $Q=\Sigma_{\beta} b_{\beta} D_{x}^{\beta}$ of $\mathcal{E}$, we define the composition $P \circ Q$ by

$$
P \circ Q=\sum_{\alpha, \beta \in Z \times N^{r-1}, \gamma \in N^{r}}\left(\begin{array}{l}
\alpha \\
\gamma
\end{array}\right) a_{\alpha} b_{\beta}^{(r)} D_{x}^{\alpha+\beta-\gamma},
$$

where $b_{\beta}^{(\gamma)}=D_{x}^{r}\left(b_{\beta}\right)$. The ring $\mathcal{E}$ has an increasing filtration by subspaces $\{\mathcal{E}(m)\}_{m \in Z}$. We have

$$
\mathcal{E}(m) \mathcal{E}(n)=\mathcal{E}(n+m) .
$$

For any $\mathcal{O}$-submodule $\mathcal{L}$ of $\mathcal{E}$ we define the induced filtration $\{\mathcal{L}(m)\}_{m \in Z}$ of $\mathcal{L}$ by $\mathcal{L}(m)=\mathcal{L} \cap \mathcal{E}(m)$.

Let $\mathcal{E}_{\phi}$ be the $\mathcal{O}$-module consisting of the formal microdifferential operators of the form

$$
\sum_{\alpha_{0}<0} a_{\alpha}(x) D_{x}^{\alpha} .
$$

The ring $\mathcal{E}$ is the direct sum of $\mathscr{D}$ and $\mathcal{E}_{\phi}$. For any $P \in \mathcal{E}$, we define $P_{+} \in \mathscr{D}$ and $P_{-} \in \mathcal{E}_{\phi}$ by the decomposition of $\mathcal{E}$ :

$$
\begin{aligned}
& \mathcal{E}=\mathscr{D} \oplus \mathcal{E}_{\phi} \\
& P=P_{+}+P_{-} .
\end{aligned}
$$

For any $\mathcal{O}$-submodule $\mathcal{L}$ of $\mathcal{E}$ we define the $\mathcal{O}$-module $\mathcal{L}$ - by $\mathcal{L}_{-}=\mathcal{L} \cap \mathcal{E}_{\phi}$. Remark that $\mathcal{E}(0)=\mathcal{O} \oplus \mathcal{E}_{\phi}(0)$.

In the following we shall study a left $\mathscr{D}$-submodule $I$ of $\mathcal{E}$ which satisfies the following condition:

$$
\mathcal{E}(m)=I(m) \oplus \mathcal{E}_{\phi}(m) \quad \text { for any } \quad m \in \boldsymbol{Z} .
$$

For example $I=\mathscr{D}$ satisfies (1.1). We make clear the structure of such a $\mathscr{D}$ submodule $I$.

Lemma 1.1. Suppose that a $\mathscr{D}$-submodule $I$ of $\mathcal{E}$ satisfies the condition (1.1). Then $I$ is generated as $\mathscr{D}$-module by a unique operator $W$ such that

$$
W \in \mathcal{E}(0) \text { and } W_{+}=1 .
$$

Proof. The operator $W$ is obtained by decomposing the identity operator $1 \in \mathcal{E}(0)$ into the sum of an operator in $I(0)$ and an operator in $\mathcal{E}_{\phi}(0)$ according to the condition (1.1): 


$$
\begin{gathered}
\mathcal{E}(0)=I(0) \oplus \mathcal{E}_{\phi}(0) \\
1=W+U .
\end{gathered}
$$

It is evident that $W$ is contained in $\mathcal{E}(0)$ and that $W_{+}=1$. Since we have

$$
\mathcal{E}(m) W=\mathcal{E}(m), \quad \mathcal{E}_{\phi}(m) W=\mathcal{E}_{\phi}(m)
$$

for any $m \in \boldsymbol{Z}$, we obtain that

$$
\mathcal{E}(m)=\mathscr{D}(m) W \oplus \mathcal{E}_{\dot{\phi}}(m) \quad \text { for any } \quad m \in \mathbb{Z} .
$$

Thus the $\mathscr{D}$-module $I^{\prime}=\mathscr{D W}$ also satisfies (1.1). Because $I$ contains $I^{\prime}$ and both satisfy (1.1), $I$ coincides with $I^{\prime}$. The uniqueness is clear.

Remark that $W$ in Lemma 1.1 is invertible by (1.2).

We investigate nonlinear evolution equations according to the program of M. Sato ([9], [10]). For any $P \in \mathcal{E}$ and any $\mathscr{D}$-submodule $I_{0}$ of $\mathcal{E}$ we define the time evolution $I_{t}$ of $I_{0}$ by the following differential equations:

$$
\frac{\partial V(t)}{\partial t}+V(t) P \in I_{t} \quad \text { for any } \quad V(t) \in I_{t} .
$$

We call $P \in \mathcal{E}$ the generator of the evolution equation (1.3).

In general we cannot find any $\mathscr{D}$-submodule $I_{t}$ which solves (1.3). In this paper we shall study the case that we can find a solution $I_{t}$ of (1.3) which is a $\mathscr{D}$-module satisfying (1.1) for any $t$. Then $I_{t}$ is generated by an operator $W(t) \in \mathcal{E}(0)$ by Lemma 1.1 and we can rewrite the equation (1.3) in terms of the generator $W(t)$.

Lemma 1.2. We fix an operator $P \in \mathcal{E}$. We assume that the solution $I_{t}$ of the evolution equation (1.3) is a D-submodule which satisfies (1.1) for any $t$. Then the equation (1.3) reduces to the following equation

$$
\frac{\partial W(t)}{\partial t}+W(t) P=\left(W(t) P W(t)^{-1}\right)_{+} W(t),
$$

where the operator $W(t)$ is the generator of $I_{t}$ in Lemma 1.1.

Proof. From the equation (1.3) there exists an operator $B(t) \in \mathscr{D}$ such that

$$
\frac{\partial W(t)}{\partial t}+W(t) P=B(t) W(t)
$$

Thus we have

$$
B(t)=W(t) P W(t)^{-1}+\frac{\partial W(t)}{\partial t} W(t)^{-1} .
$$

Since the operator $W(t)$ is contained in $1+\mathcal{E}_{\dot{\phi}}$, the operator $(\partial W(t) / \partial t) W(t)^{-1}$ is contained in $\mathcal{E}_{\dot{\phi}}$. Thus we obtain that $B(t)=\left(W(t) P W(t)^{-1}\right)_{+}$. 
Remark. The equation (1.4) is rewritten as

$$
\begin{aligned}
\frac{\partial W}{\partial t} & =\left(W P W^{-1}\right)_{+} W-W P \\
& =-\left(W P W^{-1}\right)_{-} W .
\end{aligned}
$$

The evolution equation (1.4) is associated with an infinitesimal action $\rho$ of $\mathcal{E}$ on the space $\mathcal{E}$. For $P \in \mathcal{E}$ the vector field $\rho(P)$ is given as follows.

$$
W \longrightarrow-\left(W P W^{-1}\right)_{-} W \in T_{W} \mathcal{E},
$$

where the tangent space $T_{W} \mathcal{E}$ is identified with $\mathcal{E}$ by the structure of vector space of $\mathcal{E}$.

Theorem 1.3. For any $P, Q \in \mathcal{E}$ we have

$$
\rho([P, Q])=-[\rho(P), \rho(Q)] .
$$

Proof. We denote by $\varepsilon_{1}$ and $\varepsilon_{2}$ the time parameters with respect to $P$ and $Q$, respectively. We set $\tilde{P}=I W P W^{-1}$ and $\widetilde{Q}=W Q W^{-1}$. We have

$$
\begin{aligned}
& \exp \left(\varepsilon_{1} \rho(P)\right) W \equiv\left(1-\varepsilon_{1}(\tilde{P})_{-}\right) W \bmod \varepsilon_{1}^{2}, \\
& \exp \left(\varepsilon_{2} \rho(Q)\right) W \equiv\left(1-\varepsilon_{2}(\tilde{Q})_{-}\right) W \bmod \varepsilon_{2}^{2} .
\end{aligned}
$$

Hence we have, modulo $\varepsilon_{1}^{2}, \varepsilon_{2}^{2}$

$$
\begin{aligned}
& \exp \left(\varepsilon_{1} \rho(P)\right)\left(1-\varepsilon_{2}\left(W Q W^{-1}\right)_{-}\right) \\
& \quad \equiv 1-\varepsilon_{2}\left(\left(1-\varepsilon_{1} \tilde{P}_{-}\right) \tilde{Q}_{\left.\left(1+\varepsilon_{1} \tilde{P}_{-}\right)\right)_{-}}\right. \\
& \quad \equiv 1-\varepsilon_{2} \tilde{Q}_{-}+\varepsilon_{1} \varepsilon_{2}\left[\tilde{P}_{-}, \tilde{Q}\right]_{-} .
\end{aligned}
$$

Thus we obtain, modulo $\varepsilon_{1}^{2}, \varepsilon_{2}^{2}$

$$
\begin{aligned}
\exp & \left(\varepsilon_{1} \rho(P)\right) \exp \left(\varepsilon_{2} \rho(Q)\right) W^{J} \\
& \equiv \exp \left(\varepsilon_{1} \rho(P)\right)\left(1-\varepsilon_{2}\left(W Q W^{-1}\right)_{-}\right) W \\
& \equiv\left(1-\varepsilon_{2} \tilde{Q}_{-}+\varepsilon_{1} \varepsilon_{2}\left[\tilde{P}_{-}, \tilde{Q}_{-}\right)\left(1-\varepsilon_{1} \tilde{P}_{-}\right) W^{J}\right. \\
& \equiv\left(1-\varepsilon_{1} \tilde{P}_{-}-\varepsilon_{2} \tilde{Q}_{-}+\varepsilon_{1} \varepsilon_{2}\left(\left[\tilde{P}_{-}, \tilde{Q}\right]_{-}+\tilde{Q}_{-} \tilde{P}_{-}\right)\right) W
\end{aligned}
$$

Similarly we have, modulo $\varepsilon_{1}^{2}, \varepsilon_{2}^{2}$

$$
\begin{aligned}
& \exp \left(\varepsilon_{2} \rho(Q)\right) \exp \left(\varepsilon_{1} \rho(P)\right) W \\
& \quad \equiv\left(1-\varepsilon_{2} \tilde{Q}_{-}-\varepsilon_{1} \tilde{P}_{-}+\varepsilon_{1} \varepsilon_{2}\left(\left[\tilde{Q}_{-}, \hat{P}\right]_{-}+\tilde{P}_{-} \tilde{Q}_{-}\right)\right) W .
\end{aligned}
$$

By the formula

$$
\begin{gathered}
\exp \left(\varepsilon_{1} \rho(P)\right) \exp \left(\varepsilon_{2} \rho(Q)\right) W-\exp \left(\varepsilon_{2} \rho(Q)\right) \exp \left(\varepsilon_{1} \rho(P)\right) W^{\prime} \\
\quad \equiv \varepsilon_{1} \varepsilon_{2}[\rho(P), \rho(Q)] W \bmod \varepsilon_{1}^{2}, \varepsilon_{2}^{2},
\end{gathered}
$$


we obtain

$$
\begin{aligned}
{[\rho(P), \rho(Q)] W } & =\left\{\left[\tilde{P}_{-}, \tilde{Q}_{-}+\tilde{Q}_{-} \tilde{P}_{-}-\left[\tilde{Q}_{-}, \tilde{P}_{-}-\tilde{P}_{-} \tilde{Q}_{-}\right\} W\right.\right. \\
& =\left\{\left[\tilde{P}_{-}, \tilde{Q}_{+}+\tilde{Q}_{-}\right]_{-}-\left[\tilde{Q}_{-}, \tilde{P}_{+}+\tilde{P}_{-}\right]_{-}+\left[\tilde{Q}_{-}, \tilde{P}_{-}\right]\right\} W
\end{aligned}
$$

Since $\left[\tilde{P}_{+}, \tilde{Q}_{+}\right]_{-}=0$, the right hand side of $(1.6)$ is equal to

$$
[\tilde{P}, \tilde{Q}]-W=-\rho([P, Q]) W .
$$

When $P, Q \in \mathcal{E}$ commute with each other, the following equations are compatible by Theorem 1.3 :

$$
\begin{aligned}
& \frac{\partial W}{\partial s}=\left(W P W^{-1}\right)_{+} W-W P, \\
& \frac{\partial W}{\partial t}=\left(W Q W^{-1}\right)_{+} W-W Q,
\end{aligned}
$$

where $W=W(s, t)$.

In the case $r=1$, for any $P \in \mathcal{E}$ and for any $I_{0}=\mathscr{D W}(0)$ the solution $I_{t}$ of the equation (1.3) satisfies the condition (1.1) for any $t$. With the choice $P=$ $D_{0}^{n}(n=1,2, \cdots)$, we obtain the KP-hierarchy ([7], [8]):

$$
\frac{\partial W(t)}{\partial t}+W(t) D_{0}^{n}=\left(W(t) D_{0}^{n} W(t)^{-1}\right)_{+} W(t)
$$

In higher dimensional case, we must choose a nice pair of the generator $P$ and $I_{0}=\mathscr{D W}(0)$ in order that $I_{t}$ satisfies the condition (1.1) for any $t$. We shall see the evolution equation (1.4) constrains the initial value $W(0)$ in the following example.

Example 1.1. We consider (1.4) in the case $r=2$. We take $D_{0}^{2}$ as the generator of the equation (1.4). We write

$$
W(t)=\sum_{i+j \geqq 0, j \geq 0} w_{i, j} D_{0}^{i} D_{1}^{j}, \quad w_{0,0} \equiv 1 .
$$

The operator $W(t) D_{0}^{2}$ is decomposed into the sum

Then we have

$$
\begin{aligned}
\mathcal{E} & =\mathscr{D W} \oplus \mathcal{E}_{\phi} \\
W(t) D_{0}^{2} & =\left(W(t) D_{0}^{2} W(t)^{-1}\right)_{+} W(t)+U .
\end{aligned}
$$

$$
\begin{aligned}
& \left(W(t) D_{0}^{2} W(t)^{-1}\right)_{+}=\left(D_{0}^{2}-2 \frac{\partial w_{-1,1}}{\partial x_{0}} D_{1}-2 \frac{\partial w_{-1,0}}{\partial x_{0}}\right), \\
& U=\sum_{i+j \leqq 1, i<0} u_{i, j}(x) D_{0}^{i} D_{1}^{j} \\
& =\sum_{i+j \leqq 1, i<0}\left(-2 \frac{\partial w_{i-1, j}}{\partial x_{0}}+2 \frac{\partial w_{-1,1}}{\partial x_{0}} w_{i, j-1}-\frac{\partial^{2} w_{i, j}}{\partial x_{0}^{2}}\right. \\
& \left.\quad 2 \frac{\partial w_{-1,0}}{\partial x_{0}} w_{i, j}+2 \frac{\partial w_{-1,1}}{\partial x_{0}} \frac{\partial w_{i, j}}{\partial x_{1}}\right) D_{0}^{i} D_{1}^{i} .
\end{aligned}
$$


The equation (1.4) is equivalent to the following:

$$
\begin{gathered}
\frac{\partial w_{i, j}}{\partial t}+u_{i, j}=0 \quad \text { for } \quad i+j \leqq 0, \\
\frac{\partial w_{i-1, j}}{\partial x_{0}}-\frac{\partial w_{-1,1}}{\partial x_{0}} w_{i, j-1}=0, \quad \text { for } \quad i+j=1 .
\end{gathered}
$$

The second equation constrains the initial data $W(0)$.

\section{§ 2. Integrable Systems in Higher Dimensions}

In the example 1.1 we have considered the equation (1.3) for one generator $P=D_{0}^{2}$. In this section we will introduce a space $V$ of generators, and determine the space of $\mathscr{D}$-submodules $I$ of $\mathcal{E}$ such that the condition (1.1) is preserved under the time evolution (1.3) for any $P \in V$.

First we review two known examples, the self-dual Yang-Mills equations and the self-dual Einstein equations. We can interpret both of the equations as integrable systems of three variables (see [14]).

Example 2.1. Self-dual Yang-Mills equations (see [5], [11]). The self-dual Yang-Mills equations are written in the following form

$$
\begin{gathered}
\frac{\partial A_{1}}{\partial x_{2}}\left(x_{1}, x_{2}, s, t\right)=\frac{\partial A_{2}}{\partial x_{1}}\left(x_{1}, x_{2}, s, t\right), \\
{\left[\frac{\partial}{\partial s}+A_{1}\left(x_{1}, x_{2}, s, t\right), \frac{\partial}{\partial t}+A_{2}\left(x_{1}, x_{2}, s, t\right)\right]=0}
\end{gathered}
$$

for gauge fields $A_{1}, A_{2} \in \operatorname{Mat}(n \times n)$ on four-dimensional manifolds.

The evolution equation (1.4) is generalized to the case that $W$ and $P$ have matrix coefficients. We introduce the space $\mathscr{W}_{Y M}(n)$ and the Lie algebra $V_{Y M}(n)$ :

$$
\begin{gathered}
\mathscr{W}_{Y^{\prime} M}(n)=\left\{W\left(x_{1}, x_{2}, D_{0}\right)=\sum_{i \in N} w_{i}\left(x_{1}, x_{2}\right) D_{0}^{-i} ;\right. \\
\left.w_{i} \in \operatorname{Mat}\left(n \times n, C\left[\left[x_{1}, x_{2}\right]\right]\right), w_{0} \equiv 1_{n}\right\} . \\
V_{Y M}(n)=\left\{F_{1}\left(x_{1}, x_{2}, D_{0}\right) D_{1}+F_{2}\left(x_{1}, x_{2}, D_{0}\right) D_{2}+E\left(x_{1}, x_{2}, D_{0}\right) ;\right. \\
\left.F_{1}, F_{2} \in \operatorname{Mat}(n \times n, \mathscr{D}), E \in \operatorname{Mat}(n \times n, \mathcal{E})\right\} .
\end{gathered}
$$

The evolution equation (1.4) for $P \in V_{Y M}(n)$ with any initial value $W \in \mathscr{T V}_{Y M}(n)$ has a solution in $\mathscr{W}_{Y M}(n)$. We consider the equation (1.4) for $P=D_{0} D_{1}, D_{0} D_{2} \Subset$ $V_{Y M}(n)$ :

$$
\begin{aligned}
& \frac{\partial W}{\partial s}+W D_{0} D_{1}=\left(W D_{0} D_{1} W^{-1}\right)_{+} W, \\
& \frac{\partial W}{\partial t}+W D_{0} D_{2}=\left(W D_{0} D_{2} W^{-1}\right)_{+} W .
\end{aligned}
$$

In terms of the coefficients $w_{j}$ of $W$ the equation (2.2) is written in the form 


$$
\begin{aligned}
& \frac{\partial w_{\imath}}{\partial s}=\frac{\partial w_{\imath+1}}{\partial x_{1}}-\frac{\partial w_{1}}{\partial x_{1}} w_{\imath}, \\
& \frac{\partial w_{\imath}}{\partial t}=\frac{\partial w_{\imath+1}}{\partial x_{2}}-\frac{\partial w_{1}}{\partial x_{2}} w_{\imath},
\end{aligned}
$$

for $i>0$. We set $A_{j}=\partial w_{1} / \partial x_{j}(j=1,2)$. By eliminating $w_{2}$ from the equation (2.3) for $i=1$, we obtain the equation (2.1).

Example 2.2. Self-dual Einstein equations (see [2], [12]). The self-dual Einstein equations are written in the form (see [6])

$$
\begin{gathered}
\frac{\partial A_{1}}{\partial x_{2}}\left(x_{1}, x_{2}, s, t\right)=\frac{\partial A_{2}}{\partial x_{1}}\left(x_{1}, x_{2}, s, t\right), \\
\frac{\partial B_{1}}{\partial x_{2}}\left(x_{1}, x_{2}, s, t\right)=\frac{\partial B_{2}}{\partial x_{1}}\left(x_{1}, x_{2}, s, t\right), \\
{\left[\frac{\partial}{\partial s}+A_{1}\left(x_{1}, x_{2}, s, l\right) \frac{\partial}{\partial x_{1}}+B_{1}\left(x_{1}, x_{2}, s, t\right) \frac{\partial}{\partial x_{2}},\right.} \\
\left.\frac{\partial}{\partial t}+A_{2}\left(x_{1}, x_{2}, s, t\right) \frac{\partial}{\partial x_{1}}+B_{2}\left(x_{1}, x_{2}, s, t\right) \frac{\partial}{\partial x_{2}}\right]=0, \\
\frac{\partial B_{3}}{\partial x_{2}}\left(x_{1}, x_{2}, s, t\right)+\frac{\partial A_{3}}{\partial x_{2}}\left(x_{1}, x_{2}, s, t\right)=0 \quad(j=1,2) .
\end{gathered}
$$

In the following we forget the last equation in (2.4) for simplicity.

We introduce the space $\mathscr{W}_{E}$ and the Lie algebra $V_{E}$ :

$$
\begin{aligned}
& \mathscr{W}_{E}=\left\{W=\sum_{\jmath, k \in N} \frac{1}{j ! k !} G_{1}^{\jmath} G_{2}^{k} D_{1}^{j} D_{2}^{k} ; G_{2}=\sum_{\jmath<0} g_{\imath, \jmath}\left(x_{1}, x_{2}\right) D_{0}^{\jmath}(i=1,2)\right\} . \\
& V_{E}=\left\{F_{1}\left(x_{1}, x_{2}, D_{0}\right) D_{1}+F_{2}\left(x_{1}, x_{2}, D_{0}\right) D_{2} ; F_{1}, F_{2} \in \mathcal{E}\right\} .
\end{aligned}
$$

The evolution equation (1.4) for $P \in V_{E}$ with any initial value $W \in \mathscr{W}_{E}$ has a solution in $\mathscr{W}_{E}$. We consider (1.4) for $P=D_{0} D_{1}, D_{0} D_{2} \Subset V_{E}$ :

$$
\begin{aligned}
& \frac{\partial W}{\partial s}+W D_{0} D_{1}=\left(W D_{0} D_{1} W^{-1}\right)_{+} W, \\
& \frac{\partial W}{\partial t}+W D_{0} D_{2}=\left(W D_{0} D_{2} W^{-1}\right)_{+} W .
\end{aligned}
$$

In terms of the coefficients $g_{\imath,}$ the equation (2.5) is written in the form:

$$
\begin{aligned}
& \frac{\partial g_{i, j}}{\partial s}=\frac{\partial g_{i, j-1}}{\partial x_{1}}-\frac{\partial g_{1,-1}}{\partial x_{1}} \frac{\partial g_{i, j}}{\partial x_{1}}-\frac{\partial g_{2,-1}}{\partial x_{1}} \frac{\partial g_{i, j}}{\partial x_{2}} \\
& \frac{\partial g_{i, j}}{\partial t}=\frac{\partial g_{i, j-1}}{\partial x_{2}}-\frac{\partial g_{1,-1}}{\partial x_{2}} \frac{\partial g_{i, j}}{\partial x_{1}}-\frac{\partial g_{2,-1}}{\partial x_{2}} \frac{\partial g_{i, j}}{\partial x_{2}} \text { for } i=1,2, j<0 .
\end{aligned}
$$


We set $A_{\jmath}=-\partial g_{1,-1} / \partial x_{\jmath}, B=-\partial g_{2,-1} / \partial x_{\jmath}(j=1,2)$. By eliminating $g_{2,-2}(i=1,2)$ from the equation (2.6) for $i=1,2, j=-1$, we obtain the equation (2.4).

We shall unify these two examples and obtain more general systems. We introduce the Lie subalgebra $V$ of $\mathcal{E}$ which contains both the Lie algebras $V_{Y M}$ and $V_{E}$ :

$$
\begin{array}{r}
V=\left\{F_{0}\left(x^{\prime}, D_{0}\right) x_{0}+\sum_{0<j<r} F_{\jmath}\left(x^{\prime}, D_{0}\right) D_{\jmath}+E\left(x^{\prime}, D_{0}\right) ;\right. \\
\left.F_{k}\left(x^{\prime}, D_{0}\right), E\left(x^{\prime}, D_{0}\right) \Xi \mathcal{E},(0 \leqq k<r)\right\},
\end{array}
$$

where $x^{\prime}=\left(x_{1}, x_{2}, \cdots, x_{r-1}\right)$.

Lemma 2.1. $V$ is isonor phic to $\mathscr{D}(1) \otimes_{\mathcal{O}} C[[x]]\left[x_{0}^{-1}\right]$ as a Lie algebra.

Proof. Set $X=\mathbb{C}^{r}=\left\{\left(z_{0}, z_{1}, \cdots, z_{r-1}\right) \in \mathbb{C}^{r}\right\}$, and $Y=\left\{z \subseteq X ; z_{0}=0\right\}$. We take the transformation

$$
x_{0}=z_{0}^{2} D_{z_{0}}, D_{x_{0}}=z_{0}^{-1}, \quad x^{\prime}=z^{\prime}, \quad D_{x^{\prime}}=D_{z^{\prime}} .
$$

Then the transform of $V$ is $\mathscr{D}(1) \otimes C[[z]]\left[z_{0}^{-1}\right]$.

The Lie algebra $\mathscr{D}(1) \otimes C[[x]]\left[x_{0}^{-1}\right]$ is the direct sum of the Lie algebra $\Theta \otimes_{0} C[[x]]\left[x_{0}^{-1}\right]$ of vector fields and the commutative Lie algebra $\mathbb{C}[[x]]\left[x_{0}^{-1}\right]$, where the Lie algebra $\Theta$ is defined by

$$
\Theta=\sum_{0 \leqq j<r} O D_{\jmath} .
$$

The Lie algebra $\Theta \otimes_{0} C[[x]]\left[x_{0}^{-1}\right]$ corresponds to the infinitesimal coordinate transformations, and the Lie algebra $C[[x]]\left[x_{0}^{-1}\right]$ corresponds to the infinitesimal gauge transformations of a line bundle.

Now we shall determine the set of $\mathscr{D}$-submodules in $\mathcal{E}$ such that the condition (1.1) is preserved under (1.3) with respect to any $P \subseteq V$. We introduce the subspaces $\mathscr{W}_{m}$ and $\mathscr{W}_{Y M}$ of $\mathcal{E}$ :

$$
\begin{aligned}
& W_{m}=\left\{W\left(x, D_{x}\right)=\sum_{a \in N^{r}} \frac{1}{\alpha !} G^{a} x_{0}^{a_{0}} D_{x}^{a^{\prime} \in \mathcal{E} ;}\right. \\
& \left.G^{\alpha}=G_{0}^{a_{0}} G_{1}^{a_{1}} \cdots G_{r-1}^{\alpha_{\underline{1}}-1} \text { where } G_{\jmath}=G_{\jmath}\left(x^{\prime}, D_{0}\right) \in \mathcal{E}(-1)\right\} \text {, } \\
& \mathscr{W}_{Y M}=\left\{W\left(x^{\prime}, D_{0}\right)=\sum_{\imath \in N} w_{\imath}\left(x^{\prime}\right) D_{0}^{-i} ; w_{\imath} \in \boldsymbol{C}\left[\left[x_{1}, \cdots, x_{r-1}\right]\right], w_{0} \equiv 1\right\} \text {. }
\end{aligned}
$$

Proposition 2.2. Let $I_{0}=\mathscr{D} W_{0}$ be a $\mathscr{D}$-submodule of $\mathcal{E}$. Assume that the time evolution of $I_{0}$ for any $P \in V$ also satisfies the condition (1.1). Then $W_{0}$ factorizes into the product of $W_{m} \subseteq \mathscr{W}_{m}$ and $W_{Y M} \in \mathscr{W}_{Y M}$;

$$
W_{0}=W_{m} W_{Y M} \text {. }
$$

Remark. The operator $W_{m}$ corresponds to the equation of self-dual metrics 
(see $\S 3$ ).

Proof of Proposition 2.2.

Step 1.

Lemma 2.3. Let $W(t)$ be the solution of (1.4) for $P \in V$ with $W(0)=W_{0}$. Then $\left(W(t) P W(t)^{-1}\right)$ - is contained in $\mathcal{E}_{\dot{\phi}}(0)$.

Proof. It follows from the equation (1.5) that

$$
\frac{\partial W(t)}{\partial t} W(t)^{-1}=-\left(W(t) P W(t)^{-1}\right)_{-} .
$$

Since the operator $(\partial W(t) / \partial t) W(t)^{-1}$ is contained in $\mathcal{E}(0)$, we obtain Lemma 2.3 .

Step 2. For any $P \in \mathcal{E}$, let $\tilde{P}$ be $W_{0} P W_{0}^{-1}$.

Lemma 2.4. Suppose that $P, Q \boxminus V$ commute with each other. The operator $\left[\tilde{P}_{+}, \tilde{Q}_{-}\right]_{-}$is contained in $\mathcal{E}_{\phi}(0)$.

Proof. We consider the solution $W=W(s, t)$ of the equation (1.5) for the operators $P, Q$ :

$$
\begin{aligned}
& \frac{\partial W}{\partial s}=-\left(W P W^{-1}\right)_{-} W, \\
& \frac{\partial W}{\partial t}=-\left(W Q W^{-1}\right)_{-} W,
\end{aligned}
$$

where $W=W(s, t)$. Since $[P, Q]=0$, the system (2.7) is compatible by Theorem 1.3. It follows from Lemma 2.3 that $W P W^{-1}$ is contained in $\mathscr{D}+\mathcal{E}_{\phi}(0)$. We have

$$
\begin{aligned}
\frac{\partial}{\partial t}\left(W P W^{-1}\right) & =\frac{\partial W}{\partial t} P W^{-1}-W P W^{-1} \frac{\partial W}{\partial t} W^{-1} \\
& =-\left(W Q W^{-1}\right)_{-} W P W^{-1}+I P P W^{-1}\left(W Q W^{-1}\right)_{-} \\
& =\left[W P W^{-1},\left(W Q W^{-1}\right)_{-}\right] .
\end{aligned}
$$

Hence $\left[\tilde{P}, \tilde{Q}_{-}\right]$is contained in $\mathscr{D}+\mathcal{E}_{\phi}(0)$. Since $\left[\tilde{P}_{-}, \tilde{Q}_{-}\right]$is contained in $\mathcal{E}_{\phi}(0)$ by Lemma $2.3,\left[\tilde{P}_{+}, \tilde{Q}_{-}\right]$is contained in $\mathscr{D}+\mathcal{E}_{\phi}(0)$.

Step. 3. Recall that $\tilde{P}$ denotes $W_{0} P W_{0}^{-1}$ for any $P \in \mathcal{E}$. We define the operators $G_{i}, H_{2} \in \mathcal{E}(0 \leqq i<r)$ as follows :

$$
\begin{aligned}
& \tilde{D}_{0}=D_{0}-G_{0}\left(x, D_{x}\right), \\
& \tilde{x}_{i}=x_{i}+G_{i}\left(x, D_{x}\right) \quad \text { for } \quad i=1,2, \cdots, r-1, \\
& \tilde{x}_{0}=x_{0}+H_{0}\left(x, D_{x}\right), \\
& \tilde{D}_{i}=D_{i}+H_{i}\left(x, D_{x}\right) \quad \text { for } \quad i=1,2, \cdots, r-1 .
\end{aligned}
$$


Lemma 2.5. Assume that $W_{0}$ satisfies the condition in Proposition 2.2. The operators $G_{i}, H_{i}$ are written in the following form:

$$
\begin{aligned}
G_{i}= & G_{i}\left(x^{\prime}, D_{0}\right) \in \mathcal{E}(-1), \\
H_{i}= & \sum_{0<j<r} K_{i_{j}}\left(x^{\prime}, D_{0}\right) D_{j}+L_{i}\left(x, D_{0}\right) \quad \text { for } 0 \leqq i<r, \\
& \text { where } K_{i j}\left(x^{\prime}, D_{0}\right) \in \mathcal{E}, L_{i}\left(x, D_{0}\right) \in \mathcal{E}(-1), \\
& \text { and } x^{\prime}=\left(x_{1}, \cdots, x_{r-1}\right) .
\end{aligned}
$$

Proof. We shall show the following statement $(2.9)_{n}$ by the induction on $n$ :

$$
G_{i}=G_{i}^{\prime}+G_{i}^{\prime \prime}, H_{i}=H_{i}^{\prime}+H_{i}^{\prime \prime}
$$

for some $G_{i}^{\prime}, H_{i}^{\prime}, G_{i}^{\prime \prime}$ and $H_{i}^{\prime \prime}(0 \leqq i<r)$ such that

$$
\begin{aligned}
G_{0}^{\prime \prime}, H_{i}^{\prime \prime} \in \mathcal{E}(-n), G_{i}^{\prime \prime}, H_{0}^{\prime \prime} \leqq \mathcal{E}(-n-1) \quad \text { for } 0<i<r, \\
G_{i}^{\prime}=G_{i}^{\prime}\left(x^{\prime}, D_{0}\right) \Subset \mathcal{E}(-1), \\
H_{i}^{\prime}=\sum_{0<j<r} K_{i j}\left(x^{\prime}, D_{0}\right) D_{j}+L_{i}\left(x, D_{0}\right) \quad \text { for } 0 \leqq i<r \\
\quad \text { with } K_{i j}\left(x^{\prime}, D_{0}\right), L_{i}\left(x, D_{0}\right) \in \mathcal{E}(-1) .
\end{aligned}
$$

It is evident that the statement $(2.9)_{0}$ is true. By assuming $(2.9)_{n}$ we shall prove $(2.9)_{n+1}$.

We expand the operators :

$$
\begin{aligned}
& G_{j}^{\prime \prime}=\sum_{k \leqq 0, \alpha^{\prime} \in N^{r-1}} g_{k, \alpha^{\prime}}^{(j)} D_{0}^{k} D_{x^{\prime}}^{\alpha^{\prime}}, \\
& H_{j}^{\prime \prime}=\sum_{k \leqq 0, \alpha^{\prime} \in N^{r-1}} h_{k, \alpha^{\prime}}^{(j)} D_{0}^{k} D_{x^{\prime}}^{\alpha^{\prime}} .
\end{aligned}
$$

Since $G_{0}^{\prime \prime}$ belongs to $\mathcal{E}(-n)$, we have

$$
\begin{aligned}
\widetilde{D}_{0}^{n+2} & =\left(D_{0}-G_{0}^{\prime}-G_{0}^{\prime \prime}\right)^{n+2} \\
& \equiv\left(D_{0}-G_{0}^{\prime}\right)^{n+2}-(n+2) G_{0}^{\prime \prime}\left(D_{0}-G_{0}^{\prime}\right)^{n+1} \\
& \equiv\left(D_{0}-G_{0}^{\prime}\right)^{n+2}-(n+2) G_{0}^{\prime \prime} D_{0}^{n+1} \text { modulo } \mathcal{E}(0) .
\end{aligned}
$$

The operator $\left(D_{0}-G_{0}^{\prime}\right)^{n+2}$ belongs to $\mathscr{D}+\mathcal{E}(0)$, because $G_{0}^{\prime}$ does not contain $D_{j}(0<j<r)$. Hence we obtain

$$
\widetilde{D}_{0}^{n+2} \equiv-(n+2) G_{0}^{\prime \prime} D_{0}^{n+1} \text { modulo } \mathscr{D}+\mathcal{E}(0) .
$$

Since $\widetilde{D}_{0}^{n+2}$ is contained in $\mathscr{D}+\mathcal{E}(0)$ for any $n \in \boldsymbol{Z}$ by Lemma $2.3, G_{0}^{\prime \prime} D_{0}^{n+1}$ is also contained in $\mathscr{D}+\mathcal{E}(0)$. Therefore we obtain

$$
g_{-n-\left|\alpha^{\prime}\right|, \alpha^{\prime}}^{(0)}=0 \quad \text { for } \quad\left|\alpha^{\prime}\right| \geqq 2 .
$$

Similarly we have, modulo $\mathscr{D}+\mathcal{E}(0)$ 


$$
\begin{aligned}
& \tilde{D}_{0}^{n+1} \widetilde{D}_{\jmath} \equiv-(n+1) G_{0}^{\prime \prime} D_{0}^{n} D_{j}+H_{j}^{\prime \prime} D_{0}^{n+1}, \\
& \tilde{x}_{0} \widetilde{D}_{0}^{n+2} \equiv-(n+2) x_{0} G_{0}^{\prime \prime} D_{0}^{n+1}+H_{0}^{\prime \prime} D_{0}^{n+2}, \\
& \tilde{x}_{j} \widetilde{D}_{0}^{n+1} \widetilde{D}_{k} \equiv G_{j}^{\prime \prime} D_{0}^{n+1} D_{k}+x_{\jmath}\left(H_{k}^{\prime \prime} D_{0}^{n+1}-(n+1) G_{0}^{\prime \prime} D_{0}^{n} D_{k}\right) .
\end{aligned}
$$

By Lemma 2.3, $\widetilde{D}_{0}^{n+1} \widetilde{D}_{j}, \tilde{x}_{0} \widetilde{D}_{0}^{n+2}$ and $\tilde{x}_{j} \widetilde{D}_{0}^{n+1} \widetilde{D}_{k}$ are contained in $\mathscr{D}+\mathcal{E}(0)$. Thus we obtain

$$
\begin{aligned}
& h_{-1 \alpha^{\prime} \mid-n, \alpha^{\prime}}^{(j)}=0 \quad \text { for } \quad\left|\alpha^{\prime}\right| \geqq 3, \\
& h_{-2-n, \alpha^{\prime}+e_{j}}^{(j)}=(n+1) g_{-1-n, \alpha^{\prime}}^{(0)} \quad \text { for } \quad\left|\alpha^{\prime}\right|=1, \\
& h_{-1 \alpha^{\prime} \mid-n-1, \alpha^{\prime}}^{(0)}=0 \quad \text { for } \quad\left|\alpha^{\prime}\right| \geqq 2, \\
& g_{-\left|\alpha^{\prime}\right|-n-1, \alpha^{\prime}}^{(j)}=0 \quad \text { for } \quad\left|\alpha^{\prime}\right| \geqq 2,
\end{aligned}
$$

where $j=1,2, \cdots, r-1$ and $e_{j}=(0, \cdots, \stackrel{j}{1}, \cdots, 0) \in \mathbb{N}^{r-1}$.

We denote by $\mathscr{V}$ and $\mathcal{C} \mathcal{V}^{\prime}$ the subspaces of $\mathscr{D}+\mathcal{E}(0)$ :

$$
\begin{aligned}
& \mathcal{V}=\left\{\sum_{0<k<r} F_{k}\left(x, D_{0}\right) D_{k}+E\left(x, D_{0}\right) ; F_{k}\left(x, D_{0}\right), E\left(x, D_{0}\right) \in \mathcal{E}\right\}, \\
& \mathcal{V}^{\prime}=\left\{\sum_{0<k<r} F_{k}\left(x^{\prime}, D_{0}\right) D_{k}+E\left(x, D_{0}\right) ; F_{k}\left(x^{\prime}, D_{0}\right), E\left(x, D_{0}\right) \in \mathcal{E}\right\} .
\end{aligned}
$$

For $P, Q \in C V^{\prime}$ the commutator $[P, Q]$ is contained in $\nabla$. Since $H_{\jmath}^{\prime}(0 \leqq j<r)$ are contained in $\mathcal{V}^{\prime},\left[H_{i}^{\prime}, H_{j}^{\prime}\right]$ and $\left[x_{0}, H_{j}^{\prime}\right](0 \leqq i, j<r)$ are contained in $\left.\mathscr{}\right)$.

For $j>0$ we have

$$
\begin{aligned}
{\left[\tilde{x}_{0}, \tilde{D}_{j}\right]=} & {\left[x_{0}, H_{j}\right]-\frac{\partial H_{0}}{\partial x_{j}}+\left[H_{0}, H_{j}\right] } \\
= & {\left[x_{0}, H_{j}^{\prime \prime}\right]-\frac{\partial H_{0}^{\prime \prime}}{\partial x_{j}}+\left(\left[H_{0}^{\prime \prime}, H_{j}^{\prime \prime}\right]-\dot{\top}\left[H_{0}^{\prime}, H_{j}^{\prime \prime}\right]+\left[H_{0}^{\prime \prime}, H_{j}^{\prime}\right]\right) } \\
& +\left(\left[x_{0}, H_{j}^{\prime}\right]-\frac{\partial H_{0}^{\prime}}{\partial x_{j}}+\left[H_{0}^{\prime}, H_{j}^{\prime}\right]\right) \\
\equiv & {\left[x_{0}, H_{j}^{\prime \prime}\right]-\frac{\partial H_{0}^{\prime \prime}}{\partial x_{j}} \text { modulo } \mathcal{E}(-n-2)+\mathcal{V} . }
\end{aligned}
$$

Since $\left[\tilde{x}_{0}, \tilde{D}_{j}\right]=0,\left[x_{0}, H_{j}^{\prime \prime}\right]-\left(\partial H_{0}^{\prime \prime} / \partial x_{\jmath}\right)$ belongs to $\mathcal{E}(-n-2)+C V$. By $(2.10)$ we have, modulo $\mathcal{E}(-n-2)+\mathcal{V}$

$$
\begin{aligned}
{\left[x_{0}, H_{j}^{\prime \prime}\right]-\frac{\partial H_{0}^{\prime \prime}}{\partial x_{j}} } & \\
& \equiv \sum_{\left|\alpha^{\prime}\right| \geq 2}\left(\left(n+\left|\alpha^{\prime}\right|\right) h_{-n-\left|\alpha^{\prime}\right|, \alpha^{\prime}}^{(j)}-\frac{\partial h_{-n-1-\left|\alpha^{\prime}\right|, a^{\prime}}^{(0)}}{\partial x_{j}}\right) D_{0}^{-1-n-\left|\alpha^{\prime}\right|} D_{x^{\prime}}^{\alpha^{\prime}} .
\end{aligned}
$$

We obtain from (2.12) and (2.13) that

$$
\begin{aligned}
& h_{-2-n, \alpha^{\prime}}^{(j)}=0 \quad \text { for } \quad\left|\alpha^{\prime}\right|=2, \quad 0<j<r, \\
& g_{-1-n, \alpha^{\prime}}^{(0)}=0 \text { for }\left|\alpha^{\prime}\right|=1 \text {. }
\end{aligned}
$$


Similarly by the equations

$$
\begin{aligned}
& {\left[\tilde{D}_{0}, \tilde{D}_{\jmath}\right]=\frac{\partial H_{j}}{\partial x_{0}}+\frac{\partial G_{0}}{\partial x_{\jmath}}-\left[G_{0}, H_{j}\right]=0 \text { for } 0<j<r,} \\
& {\left[\tilde{D}_{0}, \tilde{x}_{j}\right]=\frac{\partial G_{j}}{\partial x_{0}}+\left[G_{\jmath}, G_{0}\right]-\left[G_{0}, x_{\jmath}\right]=0 \text { for } 0<j<r,} \\
& {\left[\tilde{D}_{0}, \tilde{x}_{0}\right]=1+\left[D_{0}, H_{0}\right]-\left[G_{0}, x_{0}\right]+\left[G_{0}, H_{0}\right]=1,}
\end{aligned}
$$

we obtain

$$
\frac{\partial h_{-1-n, e_{k}}^{(\jmath)}}{\partial x_{0}}=0, \quad \frac{\partial h_{-2-n, e_{k}}^{(0)}}{\partial x_{0}}=0, \quad \frac{\partial g_{-1-n, 0}^{(\jmath)}}{\partial x_{0}}=0
$$

For $0<i<r$ we have modulo $\mathcal{E}(-1)$

$$
\begin{aligned}
\tilde{D}_{0}^{n} \tilde{D}_{\imath} & =\left(D_{0}-G_{0}^{\prime}-G_{0}^{\prime \prime}\right)^{n}\left(D_{\imath}+H_{i}^{\prime}+H_{\imath}^{\prime \prime}\right) \\
& \equiv\left(\left(D_{0}-G_{0}^{\prime}\right)^{n}-n G_{0}^{\prime \prime}\left(D_{0}-G_{0}^{\prime}\right)^{n-1}\right)\left(D_{\imath}+H_{i}^{\prime}+H_{\imath}^{\prime \prime}\right) \\
& \equiv-n G_{0}^{\prime \prime} D_{0}^{n-1} D_{\imath}+\left(D_{0}-G_{0}^{\prime}\right)^{n}\left(D_{\imath}+H_{i}^{\prime}\right)+H_{\imath}^{\prime \prime} D_{0}^{n} .
\end{aligned}
$$

By (2.12) and (2.14) we have

$$
\begin{aligned}
H_{\imath}^{\prime \prime} D_{0}^{n} & \equiv \sum_{0<j<r} h_{-1-n, e_{\jmath}}^{(\imath)} D_{0}^{-1} D_{\jmath} \text { modulo } \mathcal{E}(-1), \\
-n G_{0}^{\prime \prime} D_{0}^{n-1} D_{\imath} & \equiv-n g_{-n, 0}^{(0)} D_{0}^{-1} D_{\imath} \text { modulo } \mathcal{E}(-1) .
\end{aligned}
$$

We set $K_{\imath}$, and $L_{\imath}$ in $(2.9)_{n}$ as follows :

$$
\begin{gathered}
K_{\imath \jmath}\left(x^{\prime}, D_{0}\right)=\sum_{m<0} k_{\imath, j, m}\left(x^{\prime}\right) D_{0}^{m}, \\
L_{\imath}\left(x, D_{0}\right)=\sum_{m<0} l_{i, m}\left(x^{\prime}\right) D_{0}^{m} .
\end{gathered}
$$

By $(2.9)_{n}$ we have

$$
\begin{aligned}
\left(\widetilde{D}_{0} \tilde{D}_{\imath}\right)_{+} & =\left(D_{0}-G_{0}\right)\left(D_{\imath}+H_{\imath}^{\prime}+H_{\imath}^{\prime \prime}\right) \\
& =D_{0} D_{i}+\left\{D_{0}\left(H_{i}^{\prime}+\sum_{1 \leqq \jmath<r} K_{\imath \jmath}+L_{\imath}\right)\right\}_{+} \\
& =D_{0} D_{\imath}+\sum_{1 \leqq \jmath<r}\left(h_{-1, e_{\jmath}}^{(\imath)}+k_{\imath, \jmath,-1}\right) D_{\jmath}+l_{\imath,-1} .
\end{aligned}
$$

Sublemma. We have

$$
\frac{\partial g_{-n, 0}^{(0)}}{\partial x_{0}}=0
$$

Proof. The commutator $\left[\left(\tilde{D}_{0} \tilde{D}_{\imath}\right)_{+}, \tilde{D}_{0}^{n} \tilde{D}_{\imath}\right]$ is contained in $\mathscr{D}+\mathcal{E}(0)$ by Lemma 2.4. By (2.16) and (2.17) we have, modulo $\mathcal{E}(0)$

$$
\begin{aligned}
& {\left[\left(\widetilde{D}_{0} \widetilde{D}_{\imath}\right)_{+}, \tilde{D}_{0}^{n} \tilde{D}_{\imath}\right]} \\
& \quad \equiv\left[\left(\widetilde{D}_{0} \widetilde{D}_{\imath}\right)_{+},-n g_{-n, 0}^{(0)} D_{0}^{-1} D_{i}+\sum_{0<\jmath<r} h_{-1-n, e_{j}}^{(\imath)} D_{0}^{-1} D_{\jmath}+\left(D_{0}-G_{0}^{\prime}\right)^{n}\left(D_{i}+H_{i}^{\prime}\right)\right]
\end{aligned}
$$




$$
\begin{aligned}
\equiv & {\left[D_{0}, D_{i},-n g_{-n, 0}^{(0)} D_{0}^{-1} D_{i}\right] } \\
& +\left[\left(\widetilde{D}_{0} \widetilde{D}_{i}\right)_{+}, \sum_{0<j<r} h_{-1-n, e_{j}}^{(i)} D_{0}^{-1} D_{j}+\left(D_{0}-G_{0}^{\prime}\right)^{n}\left(D_{i}+H_{i}^{\prime}\right)\right] .
\end{aligned}
$$

Since $\left(\tilde{D}_{0} \widetilde{D}_{i}\right)_{+}$and $\sum_{0<j<r} h_{-1-n, e_{j}}^{(i)} D_{0}^{-1} D_{j}+\left(D_{0}-G_{0}^{\prime}\right)^{n}\left(D_{i}+H_{i}^{\prime}\right)$ are contained in $C V$, the operator

$$
\begin{aligned}
& {\left[D_{0} D_{i},-n g_{-n, 0}^{(0)} D_{0}^{-1} D_{i}\right]} \\
& \quad=-n \frac{\partial g_{-n, 0}^{(0)}}{\partial x_{0}} D_{0}^{-1} D_{\imath}^{2}-n \frac{\partial g_{-n, 0}^{(0)}}{\partial x_{i}} D_{i}-n \frac{\partial^{2} g_{-n, 0}^{(0)}}{\partial x_{0} \partial x_{\imath}}
\end{aligned}
$$

is contained in $\mathscr{D}+\mathcal{E}(0)$. This implies (2.18).

It follows from (2.11), (2.12) and (2.14) that we have

$$
\begin{gathered}
G_{0}^{\prime}-g_{-n, 0}^{(0)} D_{0}^{-n} \in \mathcal{E}(-n-1), \\
G_{i}^{\prime}-g_{-n-1,0}^{(i)} D_{0}^{-n-1} \in \mathcal{E}(-n-2), \\
H_{0}^{\prime}-\left(\sum_{0<k<r} h_{-n-2, e_{k}}^{(0)} D_{0}^{-n-2} D_{k}+h_{-n-1,0}^{(0)} D_{0}^{-n-1}\right) \subseteq \mathcal{E}(-n-2), \\
H_{i}^{\prime}-\left(\sum_{0<k<r} h_{-n-1, e_{k}}^{(i)} D_{0}^{-n-1} D_{k}+h_{-n, 0}^{(i)} D_{0}^{-n}\right) \in \mathcal{E}(-n-1),
\end{gathered}
$$

for $0<i<r$. Thus we obtain $(2.9)_{n+1}$ from (2.15) and (2.18).

Step 4. Now we shall prove Proposition 2.2. We introduce a microdifferential operator

$$
W_{m}=\sum_{\alpha \in N^{r}} \frac{1}{\alpha !} G^{\alpha} x^{\alpha_{0}} D_{x^{\prime}}^{\alpha^{\prime}},
$$

where $G^{\alpha}=G_{0}^{\alpha} G_{1}^{\alpha} \cdots G_{r-1}^{\alpha} \underline{r}^{-1}$ is given in Lemma 2.5. Then we have

$$
\begin{aligned}
{\left[D_{0}, W_{m}\right] } & =\sum_{a \in N^{r}} \frac{\alpha_{0}}{\alpha !} G^{\alpha} x_{0}^{\alpha_{0}-1} D_{x^{\prime}}^{\alpha^{\prime}} \\
& =G_{0} \sum_{\alpha \in N^{r}} \frac{1}{\alpha !} G^{\alpha} x_{0}^{\alpha_{0}} D_{x^{\prime}}^{\alpha^{\prime}}=G_{0} W_{m} .
\end{aligned}
$$

Hence we get

$$
W_{m} D_{0} W_{m}^{-1}=D_{0}-G_{0}
$$

Similarly we obtain that

$$
W_{m} x_{j} W_{m}^{-1}=x_{j}+G_{j} \quad(1 \leqq j<r) .
$$

Set $W_{Y M}=W_{m}^{-1} W$. Then it follows from (2.8), (2.20) and (2.21) that $W_{Y M}$ commutes with $D_{0}, x_{1}, \cdots, x_{r-1}$. Therefore $W_{Y M}$ is contained in $W_{Y M}$.

Thus we have completed the proof of Proposition 2.2.

We set

$$
\mathscr{W}=\left\{W_{m} W_{Y M} ; W_{m} \in \mathscr{W}_{m}, W_{Y M} \in \mathscr{W}_{Y M}\right\}
$$


We shall investigate the structure of $\mathscr{W}, \mathscr{W}_{m}$ and $\mathscr{W}_{Y M}$.

Proposition 2.6. The spaces $\mathscr{W}, \mathscr{W}_{m}$ and $\mathscr{W}_{Y M}$ are groups by the composition of microdifferential operators, and $\mathscr{W}_{Y M}$ is a normal subgroup in $W$.

Proof. It is evident that $\mathscr{W}_{Y M}$ is an abelian group.

Let $W_{m}=\Sigma_{a}(1 / \alpha !) G^{\alpha} x_{0}^{\alpha} D_{x^{\prime}}^{\alpha^{\prime}}$ and $W_{m}^{\prime}=\Sigma_{\beta}(1 / \beta !) F^{\beta} x_{0}^{\beta_{0}} D_{x^{\prime}}^{\beta^{\prime}}$ be operators in $\mathscr{W}_{m}$. For any microdifferential operator $P\left(x^{\prime}, D_{0}\right)$, we set

$$
\tilde{P}:=W_{m} P W_{m}^{-1}=P\left(x_{1}+G_{1}, \cdots, x_{r-1}+G_{r-1}, D_{0}-G_{0}\right) .
$$

The last equality follows from (2.20) and (2.21). Noting that $\widetilde{P}$ commutes with $G_{\jmath}$, the composition

$$
\begin{aligned}
W_{m} W_{m}^{\prime} & =\sum_{\gamma} \frac{1}{\beta !} \tilde{F}^{\beta} W_{m} x_{0}^{\beta_{0}} D_{x^{\prime}}^{\beta^{\prime}} \\
& =\sum_{\alpha, \beta} \frac{1}{\beta !} \tilde{F}^{\beta} \frac{1}{\alpha !} G^{\alpha} x_{0}^{\alpha_{0}+\beta_{0}} D_{x^{\prime}}^{\alpha^{\prime}+\beta^{\prime}} \\
& =\sum_{\gamma} \frac{1}{\gamma !}(\tilde{F}+G)^{\gamma} x_{0}^{\gamma_{0}} D_{x^{\prime}}^{\gamma^{\prime}}
\end{aligned}
$$

is contained in $\mathscr{W}_{m}$. For $W_{Y M}=\sum_{i} \mathscr{W}_{2}\left(x^{\prime}\right) D_{0}^{-i} \in W_{Y M}$ the operator

$$
\begin{aligned}
& W_{m} W_{Y M} W_{m}^{-1} \\
& =\sum_{i \geqq 0} w_{i}\left(x_{1}+G_{1}, x_{2}+G_{2}, \cdots, x_{r-1}+G_{r-1}\right)\left(D_{0}-G_{0}\right)^{-i}
\end{aligned}
$$

is contained in $\mathscr{W}_{Y M}$. Since $\mathscr{W}=\mathscr{W}_{m} \mathscr{W}_{Y M}$, it follows from (2.22) and (2.23) that $\mathscr{W}$ is a group and that $\mathscr{W}_{Y M}$ is a normal subgroup of $W$.

We define the Lie subalgebras $V_{m}$ and $V_{Y M}$ of $V$

$$
\begin{gathered}
V_{m}=\left\{F_{0}\left(x^{\prime}, D_{0}\right) x_{0}+\sum_{0<j<r} F_{j}\left(x^{\prime}, D_{0}\right) D_{j} ; F_{k}\left(x^{\prime}, D_{0}\right) \in \mathcal{E},(0 \leqq k<r)\right\}, \\
V_{Y M}=\left\{E\left(x^{\prime}, D_{0}\right) ; E\left(x^{\prime}, D_{0}\right) \subseteq \mathcal{E}\right\} .
\end{gathered}
$$

We have

$$
V=V_{m} \oplus V_{Y M} \text {. }
$$

Proposition 2.7. For any $P \in V$ (resp. $V_{m}, V_{Y M}$ ) and $W \equiv \mathscr{W}$ (resp. $\mathscr{W}_{m}, W_{Y M}$ ), we have $W P W^{-1} \in V$ (resp. $\left.V_{m}, V_{Y M}\right)$.

Proof. For $I V=\Sigma_{\alpha}(1 / \alpha !) G^{\alpha} x_{0}^{\alpha} 0 D_{x^{\prime}}^{\alpha^{\prime}} € W_{m}$ we have

$$
\begin{aligned}
{\left[D_{i}, W^{\prime}\right] } & =\sum_{0 \leqq j<r} \sum_{a} \frac{\alpha_{j}}{\alpha !} G^{\alpha-\varepsilon_{j}} \frac{\partial G_{j}}{\partial x_{i}} x_{0}^{a_{0} 0} D_{x^{\prime}}^{\alpha^{\prime}} \\
& =\sum_{0<j<r} \frac{\partial G_{j}}{\partial x_{i}} W D_{j}+\frac{\partial G_{0}}{\partial x_{i}} W x_{0}
\end{aligned}
$$


where $i=1,2, \cdots, r-1$, and $\varepsilon_{\jmath}=(0, \cdots, \stackrel{j}{1}, \cdots, 0) \in \mathbb{N}^{r}$. Similarly we have

$$
\left[x_{0}, W\right]=\sum_{0<j<r}\left[x_{0}, G_{j}\right] W D_{j}+\left[x_{0}, G_{0}\right] W x_{0} .
$$

Hence we obtain

$$
\begin{aligned}
& I V D_{\imath} W^{-1}=D_{\imath}-\sum_{0<j<r} \frac{\partial G_{3}}{\partial x_{\imath}} W D_{\jmath} W^{-1}-\frac{\partial G_{0}}{\partial x_{\imath}} W x_{0} W^{-1} \text { for } 0<i<r, \\
& W x_{0} W^{-1}=x_{0}-\sum_{0<j<r}\left[x_{0}, G_{j}\right] W D_{\jmath} W^{-1}-\left[x_{0}, G_{0}\right] W x_{0} W^{-1} .
\end{aligned}
$$

We set

$$
G_{\imath \jmath}=\left\{\begin{array}{l}
\frac{\partial G_{\jmath}}{\partial x_{2}} \quad \text { for } \quad 0<i<r, \\
{\left[x_{0}, G_{\jmath}\right] \quad i=0,}
\end{array}\right.
$$

for $0 \leqq j<r$. Then we have

$$
\begin{aligned}
& x_{0}=\left(1+G_{00}\right) W x_{0} W^{-1}+\sum_{0<j<r} G_{0 \jmath} W D_{\jmath} W^{-1}, \\
& D_{\imath}=G_{20} W x_{0} W^{-1}+\sum_{0<j<r}\left(\delta_{\imath \jmath}+G_{\imath \jmath}\right) W D_{\jmath} W^{-1} \quad \text { for } 0<i<r .
\end{aligned}
$$

Since $G_{\imath \jmath} \in \mathcal{E}(-1)$, the matrix $\left(\delta_{\imath \jmath}+G_{\imath \jmath}\right)_{0 \leqq i, \jmath<r}$ is invertible. Hence $W D_{\imath} W^{-1}$ $(0<i<r)$ and $W x_{0} W^{-1}$ are contained in $V_{m}$, because $G_{\imath}$ is independent of $x_{0}$, independent of $x_{0}, D_{1}, \cdots D_{r-1}$. For any operator $P=P\left(x^{\prime}, D_{0}\right) \in \mathcal{E}, W P W^{-1}$ is independent of $x_{0}, D_{1}, \cdots, D_{r-1}$ by (2.20) and (2.21). Hence we get Proposition 2.7 for $V_{m}$ and $\mathscr{W}_{m}$.

The proposition is evident for $V_{Y M}$ and $\mathscr{W}_{Y M}$. For $W_{m} \subseteq \mathscr{W}_{m}$ and $E \equiv V_{Y M}$ the operator $W_{m} E W_{m}^{-1}$ is contained in $V_{Y_{M}}$ by (2.20) and (2.21). For $W_{Y M} \in$ $\Psi_{Y Y I}$ ive have

$$
\begin{aligned}
& W_{Y M} D_{\imath} W_{Y M}^{-1}=D_{2}-\frac{\partial W_{Y M}}{\partial x_{\imath}} W_{Y M}^{-1} \quad \text { for } 0<i<r, \\
& W_{Y M} x_{0} W_{Y M}^{-1}=x_{0}-\left[x_{0}, W_{Y M}\right] W_{Y M}^{-1} .
\end{aligned}
$$

Since $W_{Y M}$ commutes with $D_{0}, x_{1}, \cdots, x_{r-1}$, the operator $W_{Y M} P V_{Y}^{-1}$ is contained in $V$ for any $P E V$. Since $V=V_{m} \oplus V_{Y M}$ and $\mathscr{W}=\mathscr{W} \mathscr{V}_{m} W_{Y M}$, we get Proposition 2.7 .

Example 2.3. We shall write down the evolution equations (1.4) for $W \in \mathscr{W}$ in the case $r=3$. We take $D_{0} D_{1}, D_{0} D_{2}$ as generators of (1.4).

We set

$$
\begin{aligned}
W= & \left(\sum_{\alpha \in N^{3}} \frac{1}{\alpha !} G^{\alpha} x_{0}^{\alpha_{0}} D_{x^{\prime}}^{a^{\prime}}\right)\left(\sum_{i} w_{\imath}\left(x^{\prime}\right) D_{0}^{-i}\right), \\
& \text { where } G_{\imath}\left(x_{1}, x_{2}, D_{0}\right)=\sum_{j<0} g_{i, \jmath}\left(x_{1}, x_{2}\right) D_{0}^{\jmath} \quad(i=1,2) .
\end{aligned}
$$

Then we have 


$$
\begin{aligned}
& \left(\widetilde{D}_{0} \tilde{D}_{1}\right)_{+}=D_{0} D_{1}-\frac{\partial g_{0,-1}}{\partial x_{1}} x_{0}-\sum_{\imath=1,2} \frac{\partial g_{\imath,-1}}{\partial x_{1}} D_{\imath}-\frac{\partial w_{1}}{\partial x_{1}} \\
& \left(\widetilde{D}_{0} \widetilde{D}_{2}\right)_{+}=D_{0} D_{2}-\frac{\partial g_{0,-1}}{\partial x_{2}} x_{0}-\sum_{i=1,2} \frac{\partial g_{\imath,-1}}{\partial x_{2}} D_{\imath}-\frac{\partial w_{1}}{\partial x_{2}} .
\end{aligned}
$$

Taking time parameters $s$ and $t$ with respect to $D_{0} D_{1}$ and $D_{0} D_{1}$, respectively, we obtain the evolution equations

$$
\begin{aligned}
& \frac{\partial W}{\partial s}+W D_{0} D_{1}=\left(D_{0} D_{1}-\frac{\partial g_{0,-1}}{\partial x_{1}} x_{0}-\sum_{\imath=1,2} \frac{\partial g_{\imath,-1}}{\partial x_{1}} D_{\imath}-\frac{\partial w_{1}}{\partial x_{1}}\right) W, \\
& \frac{\partial W}{\partial t}+W D_{0} D_{2}=\left(D_{0} D_{2}-\frac{\partial g_{0,-1}}{\partial x_{2}} x_{0}-\sum_{\imath=1,2} \frac{\partial g_{\imath,-1}}{\partial x_{2}} D_{\imath}-\frac{\partial w_{1}}{\partial x_{2}}\right) W .
\end{aligned}
$$

It follows from (2.26) that we obtain the Zakharov-Shabat type equation

$$
\begin{aligned}
& {\left[\frac{\partial}{\partial s}-D_{0} D_{1}+\frac{\partial g_{0,-1}}{\partial x_{1}} x_{0}+\sum_{i=1,2} \frac{\partial g_{\imath,-1}}{\partial x_{1}} D_{\imath}+\frac{\partial w_{1}}{\partial x_{1}},\right.} \\
& \left.\quad \frac{\partial}{\partial t}-D_{0} D_{2}+\frac{\partial g_{0,-1}}{\partial x_{2}} x_{0}+\sum_{i=1,2} \frac{\partial g_{\imath,-1}}{\partial x_{2}} D_{\imath}+\frac{\partial w_{1}}{\partial x_{2}}\right]=0 .
\end{aligned}
$$

We shall investigate the infinitesimal action $\rho$ of the Lie subalgebra $V$ of $\mathcal{E}$. Remark that the Lie algebra of the group $\mathscr{W}\left(\right.$ resp. $\mathscr{W}_{m}, \mathscr{W}_{Y M}$ ) is canonically isomorphic to the Lie subalgebra $V_{-}=V \cap \mathcal{E}_{\dot{\phi}}$ (resp. $\left(V_{m}\right)_{-},\left(V_{Y M}\right)_{-}$).

Theorem 2.8. The action $\rho$ of $V$ (resp. $V_{m}, V_{Y M}$ ) preserves the space $\mathscr{W}$ (resp. $\left.\mathscr{W}_{m}, \mathscr{W}_{Y M}\right)$. The action of $V_{-}$(resp. $\left.\left(V_{m}\right)_{-},\left(V_{Y M}\right)_{-}\right)$coincides with the infinitesimal right action of on the group $\left.W_{(\text {resp. }} \mathscr{W}_{m}, W_{Y M}\right)$.

Proof. For any element $P \in V$ (resp. $V_{m}, V_{Y M}$ ) and any operator $W \in \mathscr{W}$ (resp. $\mathscr{W}_{m}, \mathscr{W}_{Y M}$ ), we have

$$
\rho(P)=-\left(W P W^{-1}\right)_{-} W \in T_{W} \mathcal{E} .
$$

The tangent space $T_{W} \mathscr{W}$ (resp. $T_{W} \mathscr{W}_{m}, T_{W} \mathscr{W}_{Y M}$ ) is identified with $V_{-} W$ (resp. $\left.\left(V_{m}\right)_{-} W,\left(V_{Y M I}\right)_{-} W\right)$. By Proposition 2.7, $-\left(W P W^{-1}\right)_{-} W$ is contained in $T_{W} \mathscr{W}$ (resp. $\left.T_{W} \mathscr{W}_{m}, T_{W} \mathscr{W}_{Y M}\right)$. Taking $P \in V_{-}$(resp. $\left.\left(V_{m}\right)_{-},\left(V_{Y M}\right)_{-}\right)$, we have $\rho(P)=$ $-W P$. Hence the action $\rho$ is the right action of vector fields.

By Theorem 2.8 the Lie algebra $V$ (resp. $V_{m}, V_{Y M}$ ) acts on $\mathscr{W}\left(\right.$ resp. $\mathscr{W}_{m}$, $\left.\mathscr{W}_{Y M}\right)$ transitively.

\section{$\S 3$. Twistor Theory and Integrable Systems}

On oriented Riemannian manifolds of dimension four, the Weyl curvature 
tensor $C$ decomposes into two components, the self-dual part $C_{+}$and the antiself-dual part $C_{\text {. }}$. A manifold is called self-dual (resp. anti-self-dual) when $C_{-}$ (resp. $C_{+}$) vanishes. Penrose [5] showed that the vanishing of the anti-selfdual part of the Weyl tensor is precisely the integrability condition of the existence of a curved twistor space.

In this section we prove that the equation $C_{+}=0$ is the compatibility condition of the deformation equations of filtered $\mathscr{D}$-submodules in $\mathcal{E}$ (See [13] in which the Frobenius integrability condition of the equations of self dual metrics is discussed). We get the equations of self-dual metrics from the equation (2.26) for $W \in \mathscr{W}_{m}$.

Let $M$ be a complex four-manifold and $g$ a holomorphic metric, i.e. a nondegenerate symmetric holomorphic covariant two-tensor on $M$. We shall choose a holomorphic orientation on $M$ which is necessary to define the complex Hodge *-operator. Our discussion being only local, we can assume the existence of two complex vector bundles $S_{+}$and $S_{-}$: the bundles of self-dual and anti-selfdual spinors.

Let $\left\{e_{j}\right\}_{j=1,2,3,4}$ denote a local coframe on $M$ such that $g=e_{1} e_{2}+e_{3} e_{4}$. We can write them in spinor language as

$$
\left[\begin{array}{rr}
e_{4} & e_{2} \\
-e_{1} & e_{3}
\end{array}\right]=\left[\begin{array}{ll}
\phi_{1} \phi_{1} & \phi_{1} \phi_{2} \\
\psi_{2} \phi_{1} & \phi_{2} \phi_{2}
\end{array}\right]
$$

where $\phi_{1}, \phi_{2}$ (resp. $\phi_{1}, \phi_{2}$ ) are the bases of self-dual (resp. anti-self-dual) spinor coframes.

We take $P=P\left(S_{-}\right)$, the projective bundle of the rank two vector bundle $S_{-}$. We parametrize $S_{-}$locally by

$$
\left(x, \mu_{1}, \mu_{2}\right) \longrightarrow \mu_{1} \phi_{1}(x)+\mu_{2} \phi_{2}(x),
$$

and $\mu=\mu_{1} / \mu_{2}$ is an affine coordinate for $\mu_{2} \neq 0$.

Theorem 3.1. ([5]) The Riemannian manifold $(M, g)$ is self-dual iff the following Pfaffian system $\Omega$ on $P$ is integrable:

$$
\begin{aligned}
\theta & :=d \mu+\omega_{21} \mu^{2}-\left(\omega_{22}-\omega_{11}\right) \mu-\omega_{12}=0, \\
\Omega: \sigma_{1} & :=\mu e_{4}+e_{2}=0, \\
\sigma_{2} & :=-\mu e_{1}+e_{4}=0,
\end{aligned}
$$

where $\omega_{i j}$ is the connection form of $S_{-}$with respect to the frame $\phi_{1}$ and $\phi_{2}$.

Let $A(\Omega)$ be the sheaf of vector fields orthogonal to the Pfaffian system $\Omega$. The sheaf $A(\Omega)$ is a Lie algebra iff $\Omega$ is integrable. In this case there exists a local basis $\left(v_{1}, v_{2}\right)$ of $A(\Omega)$ such that $\left[v_{1}, v_{2}\right]=0$.

Proposition 3.2. Assume $(M, g)$ is self-dual. With appropriate coordinates 
$\left(\lambda, x_{1}, x_{2}, s, t\right)$ of $P$, there exists a commuting basis $\left(v_{1}, v_{2}\right)$ of $A(\Omega)$, in the following form

$$
\begin{aligned}
& v_{1}=\frac{\partial}{\partial s}-\lambda \frac{\partial}{\partial x_{1}}-\left(\frac{\partial R}{\partial x_{1}} \frac{\partial}{\partial x_{1}}+\frac{\partial S}{\partial x_{1}} \frac{\partial}{\partial x_{2}}+\frac{\partial T}{\partial x_{1}} \frac{\partial}{\partial \lambda}\right), \\
& v_{2}=\frac{\partial}{\partial t}-\lambda \frac{\partial}{\partial x_{2}}-\left(\frac{\partial R}{\partial x_{2}} \frac{\partial}{\partial x_{1}}+\frac{\partial S}{\partial x_{2}} \frac{\partial}{\partial x_{2}}+\frac{\partial T}{\partial x_{2}} \frac{\partial}{\partial \lambda}\right),
\end{aligned}
$$

where the functions $R, S$ and $T$ do not depend on $\lambda$.

Proof. First we notice the following lemma.

Lemma 3.3. ([3]) Let $(M, g)$ be a self-dual Riemannian four-manifold. Then there exist local coordinates $\left(p_{1}, p_{2}, q_{1}, q_{2}\right)$ of $M$ such that

$$
g=\sum_{i, j=1,2} P_{i j}(p, q) d p_{i} d q_{j} .
$$

It follows from Lemma 3.3 that we can take local frames $\left\{e_{j}\right\}_{j=1,2,3,4}$ as follows:

$$
\begin{array}{ll}
e_{1}=-d p_{1}, & e_{2}=-\left(P_{11} d q_{1}+P_{12} d q_{2}\right), \\
e_{4}=d p_{2}, & e_{3}=P_{21} d q_{1}+P_{22} d q_{2} .
\end{array}
$$

By Theorem 3.1 the ideal $g$ generated by $\left(\theta, \sigma_{1}, \sigma_{2}\right)$ is closed under the exterior derivative $d$. Thns we have

$$
\begin{aligned}
& d \sigma_{1} \wedge \theta \wedge \sigma_{1} \wedge \sigma_{2}=0, \\
& d \sigma_{2} \wedge \theta \wedge \sigma_{1} \wedge \sigma_{2}=0 .
\end{aligned}
$$

By direct calculations we have

$$
\begin{aligned}
& d \sigma_{1} \wedge \theta \wedge \sigma_{1} \wedge \sigma_{2}=\left(\left(\frac{\partial P_{12}}{\partial q_{1}}-\frac{\partial P_{11}}{\partial q_{2}}\right) \mu^{2}+K \mu+L\right) d \mu \wedge d p_{1} \wedge d p_{2} \wedge d q_{1} \wedge d q_{2}, \\
& d \sigma_{2} \wedge \theta \wedge \sigma_{1} \wedge \sigma_{2}=\left(\left(\frac{\partial P_{22}}{\partial q_{1}}-\frac{\partial P_{21}}{\partial q_{2}}\right) \mu^{2}+M \mu+N\right) d \mu \wedge d p_{1} \wedge d p_{2} \wedge d q_{1} \wedge d q_{2},
\end{aligned}
$$

for functions $K, L, M$ and $N$ independent of $\mu$. Thus we have

$$
\frac{\partial P_{12}}{\partial q_{1}}-\frac{\partial P_{11}}{\partial q_{2}}=0, \quad \frac{\partial P_{22}}{\partial q_{1}}-\frac{\partial P_{21}}{\partial q_{2}}=0
$$

Hence we can define new coordinates $\left(x_{1}, x_{2}, s, t, \mu\right)$ by the following equations:

$$
\begin{gathered}
\frac{\partial x_{1}}{\partial q_{i}}=-P_{1 i}, \quad \frac{\partial x_{2}}{\partial q_{i}}=P_{2 i}, \quad(i=1,2) \\
s=p_{2}, \quad t=-p_{1} .
\end{gathered}
$$

The differential forms $\theta, \sigma_{1}$ and $\sigma_{2}$ are written in these coordinates as follows: 


$$
\begin{gathered}
\theta=d \mu+\mu^{2}\left(E_{1} e_{2}+E_{2} e_{3}\right)+\mu\left(F_{1} e_{2}+F_{2} e_{3}\right)+\sum_{j} J_{\jmath} e_{\jmath}, \\
\sigma_{1}=\mu d s+\left(d x_{1}+A_{1} d s+A_{2} d t\right), \\
\sigma_{2}=\mu d t+\left(d x_{2}+B_{1} d s+B_{2} d t\right),
\end{gathered}
$$

for functions $A_{\jmath}, B_{\jmath}, E_{\jmath}, F_{\jmath}(j=1,2)$, and $J_{\jmath}(j=1,2,3,4)$ independent of $\mu$. It is easily verified that the following vectors $v_{1}, v_{2}$ belong to $A(\Omega)$ :

$$
\begin{aligned}
& v_{1}=-\mu \frac{\partial}{\partial x_{1}}+\frac{\partial}{\partial s}-A_{1} \frac{\partial}{\partial x_{1}}-B_{1} \frac{\partial}{\partial x_{2}}+\left(E_{1} \mu^{3}+F_{1} \mu^{2}+J_{2} \mu-J_{4}\right) \frac{\partial}{\partial \mu}, \\
& v_{2}=-\mu \frac{\partial}{\partial x_{2}}+\frac{\partial}{\partial t}-A_{2} \frac{\partial}{\partial x_{1}}-B_{2} \frac{\partial}{\partial x_{2}}+\left(E_{2} \mu^{3}+F_{2} \mu^{2}+J_{3} \mu-J_{1}\right) \frac{\partial}{\partial \mu} .
\end{aligned}
$$

We set the vector field

$$
\begin{aligned}
& l_{1}=\frac{\partial}{\partial s}-A_{1} \frac{\partial}{\partial x_{1}}-B_{1} \frac{\partial}{\partial x_{2}}, \\
& l_{2}=\frac{\partial}{\partial t}-\dot{A}_{2} \frac{\partial}{\partial x_{1}}-B_{2} \frac{\partial}{\partial x_{2}} .
\end{aligned}
$$

The commutator $\left[v_{1}, v_{2}\right]$ is written in the following form

$$
\begin{aligned}
& {\left[v_{1}, v_{2}\right]=\left(E_{2} \mu^{3}+F_{2} \mu^{2}\right) \frac{\partial}{\partial x_{1}}-\left(E_{1} \mu^{3}+F_{1} \mu^{2}\right) \frac{\partial}{\partial x_{2}}} \\
& +\left\{\left(E_{2} F_{1}-E_{1} F_{2}\right) \mu^{4}+\left(2 E_{2} J_{1}-2 E_{1} J_{2}+l_{1}\left(E_{2}\right)-l_{2}\left(E_{1}\right)\right) \mu^{3}\right. \\
& \left.+\left(F_{2} J_{1}-F_{1} J_{2}+\frac{\partial J_{2}}{\partial x_{2}}-\frac{\partial J_{3}}{\partial x_{1}}+l_{1}\left(F_{2}\right)-l_{2}\left(F_{1}\right)\right) \mu^{2}\right\} \frac{\partial}{\partial \mu}+\mu u_{1}+u_{0},
\end{aligned}
$$

where the coefficients of vectors $u_{1}$ and $u_{0}$ are independent of $\mu$.

By the integrability condition, $\left[v_{1}, v_{2}\right]$ is a linear combination of $v_{1}$ and $v_{2}$ and since $\left[v_{1}, v_{2}\right]$ does not contain $(\partial / \partial s)$ nor $(\partial / \partial t), v_{1}$ and $v_{2}$ commute with each other. It follows from (3.5) that

$$
E_{\jmath}=0, \quad F_{\jmath}=0(j=1,2), \quad \frac{\partial J_{2}}{\partial x_{2}}=\frac{\partial J_{3}}{\partial x_{1}} .
$$

Thus there exists a function $f=f\left(x_{1}, x_{2}, s, t\right)$ such that

$$
\frac{\partial f}{\partial x_{1}}=J_{2}, \quad \frac{\partial f}{\partial x_{2}}=J_{3} \text {. }
$$

We can take new coordinates $\left(\lambda=\mu+f, x_{1}, x_{2}, s, t\right)$. With these coordinates we have

$$
\begin{aligned}
& v_{1}=\frac{\partial}{\partial s}-\lambda \frac{\partial}{\partial x_{1}}-\left(A_{1} \frac{\partial}{\partial x_{1}}+B_{1} \frac{\partial}{\partial x_{2}}+C_{1} \frac{\partial}{\partial \lambda}\right), \\
& v_{2}=\frac{\partial}{\partial t}-\lambda \frac{\partial}{\partial x_{2}}-\left(A_{2} \frac{\partial}{\partial x_{1}}+B_{2} \frac{\partial}{\partial x_{2}}+C_{2} \frac{\partial}{\partial \lambda}\right),
\end{aligned}
$$


where $C_{1}=(\partial f / \partial s)-\left(J_{4}+J_{2} A_{1}+J_{3} B_{1}\right)$ and $C_{2}=(\partial f / \partial t)-\left(J_{1}+J_{2} A_{2}+J_{3} B_{2}\right)$. Again taking the coefficients of $\mu$ in $\left[v_{1}, v_{2}\right]$, we obtain that

$$
\frac{\partial A_{1}}{\partial x_{2}}=\frac{\partial A_{2}}{\partial x_{1}}, \quad \frac{\partial B_{1}}{\partial x_{2}}=\frac{\partial B_{1}}{\partial x_{1}}, \quad \frac{\partial C_{1}}{\partial x_{2}}=\frac{\partial C_{1}}{\partial x_{1}} .
$$

Thus there exist functions $R, S$ and $T$ which are independent of $\lambda$ such that

$$
\frac{\partial R}{\partial x_{j}}=A_{j}, \quad \frac{\partial S}{\partial x_{j}}=B_{j}, \quad \frac{\partial T}{\partial x_{j}}=C_{j} \quad \text { for } \quad j=1,2 .
$$

This completes the proof of Proposition 3.2.

Remark. By Theorem 3.1 and Proposition 3.2 the equations of self-dual metrics are equivalent to the compatibility condition $\left[v_{1}, v_{2}\right]=0$ :

$$
\begin{gathered}
\frac{\partial^{2} T}{\partial t \partial x_{1}}-\frac{\partial^{2} T}{\partial s \partial x_{2}}-\frac{\partial R}{\partial x_{2}} \frac{\partial^{2} T}{\partial x_{1}^{2}}+\left(\frac{\partial R}{\partial x_{1}}-\frac{\partial S}{\partial x_{2}}\right) \frac{\partial^{2} T}{\partial x_{1} \partial x_{2}}+\frac{\partial S}{\partial x_{1}} \frac{\partial^{2} T}{\partial x_{2}^{2}}=0 \\
\frac{\partial^{2} R}{\partial t \partial x_{1}}-\frac{\partial^{2} R}{\partial s \partial x_{2}}-\frac{\partial T}{\partial x_{2}}-\frac{\partial R}{\partial x_{2}} \frac{\partial^{2} R}{\partial x_{1}^{2}}+\left(\frac{\partial R}{\partial x_{1}}-\frac{\partial S}{\partial x_{2}}\right) \frac{\partial^{2} R}{\partial x_{1} \partial x_{2}}+\frac{\partial S}{\partial x_{1}} \frac{\partial^{2} R}{\partial x_{2}^{2}}=0 \\
\frac{\partial^{2} S}{\partial t \partial x_{1}}-\frac{\partial^{2} S}{\partial s \partial x_{2}}+\frac{\partial T}{\partial x_{1}}-\frac{\partial R}{\partial x_{2}} \frac{\partial^{2} S}{\partial x_{1}^{2}}+\left(\frac{\partial R}{\partial x_{1}}-\frac{\partial S}{\partial x_{2}}\right) \frac{\partial^{2} S}{\partial x_{1} \partial x_{2}}+\frac{\partial S}{\partial x_{1}} \frac{\partial^{2} S}{\partial x_{2}^{2}}=0 .
\end{gathered}
$$

In the equation (2.26) we take $W \equiv W_{m}$. In this case $w_{1}$ vanishes. Replacing $g_{0,1}, g_{1,1}$ and $g_{2,1}$ with $R, S$ and $T$, respectively. The equations (2.27) reduces to (3.6). Therefore we have

Theorem 3.4. The Lie algebra $V_{m}$ acts on the space of self-dual metrics. This action is transitive.

Let $\mathscr{W}_{0}$ be the space of $W \in \mathscr{W}$ which commute with $D_{0}$. In the equation (2.26) we take $W \in \mathscr{W}_{0}$. In this case $G_{0}$ vanishes. The equation (2.27) reduces to the composed system of the self-dual Yang-Mills equations and the self-dual Einstein equations (see Examples 2.1 and 2.2). Let $V_{0}$ be the Lie subalgebra of $V$ :

$$
\begin{aligned}
V_{0}= & \left\{\sum_{0 \leqq j<r} F_{j}\left(x^{\prime}, D_{0}\right) D_{j}+E\left(x^{\prime}, D_{0}\right) ;\right. \\
& \left.F_{k}\left(x^{\prime}, D_{0}\right) \in \mathcal{E}(0 \leqq k<r), E\left(x^{\prime}, D_{0}\right) \subseteq \mathcal{E}\right\} .
\end{aligned}
$$

Then $V_{0}$ acts on $\mathscr{W}_{0}$. Thus the self-dual Einstein equations are a specialization of our integrable system.

\section{Acknowledgement}

I express my deep appreciation to Prof. M. Sato for enlightening discussions 
and useful suggestions, and also to Prof. T. Kawai for constant encouragement. I would like to show my most sincere gratitude for the help of Prof. M. Kashiwara. I wish to thank Prof. K. Takasaki and Mr. A. Nakayashiki for useful dicussions. Finally thanks are due to Prof. E. Date, Prof. M. Jimbo and Prof. T. Miwa for reading the manuscript very carefully.

\section{References}

[1] Atiyah, M.F., Hitchin, N. J. and Singer, I.M., Self-duality in four-dimensional Riemannian geometry, Proc. R. Soc. London, A. 362 (1978), 425-461.

[2] Boyer, C.P. and Plebanski, J.F., An infinite hierarchy of conservation laws and nonlinear superposition principles for self-dual Einstein spaces, J. Math. Phys., 26 (1985), 229-234.

[3] — Conformally sel-dual spaces and Maxwell's equations, Phys. Lett., 106A (1984), 125-129.

[4] Noumi, M., Wronskian determinants and Grobner representation of a linear differential equation, In "Algebraic Analysis", Academic Press (1988), 549-569.

[5] Penrose, R., Nonlinear gravitons and curved twistor theory, Gen. Rel. Grav., 7 (1976), 31-52.

[6] Plebanski, J.F., Some solutions of complex Einstein equations, J. Math. Phys., 16 (1975), 2395-2402.

[7] Sato, M., Soliton equations as dynamical systems on infinite dimensional Grassmann manifolds, RIMS Kokyuroku, (Kyoto Univ.) 439 (1986), 30-46.

[8] Sato, M. and Sato, Y., Soliton equations as dynamical systems on infinite Grassmann manifolds, Lecture Notes in Num. Appl. Anal., 5 (1982), 259-271.

[9] Sato, M., Lecture notes in Kyoto Univ. (1984-1985, written by T. Umeda, in Japanese), Surikaiseki lecture note No. 5 (1989).

[10] - The KP hierachy and infinite-dimensional Grassmann manifolds, Proc. Symp. Pure Math., 49 Part 1 (1989), 51-66,

- $\mathcal{D}$-modules and non-linear integrable systems, to appear.

[11] Takasaki, K., A new approach to the self-dual Yang-Mills equations, Commun. Math. Phys., 94 (1984), 35-59.

[12] - Aspects of integrability in self-dual Einstein metrics and related equations, Publ. RIMS, 22 (1986), 949-990.

[13] — Conformal self-dual metrics and integrability, RIMS Kokyuroku (Kyoto Univ.), 592 (1986), 30-57.

[14] - Integrable systems as deformations of $\mathscr{D}$-modules, Proc. Symp. Pure Math., 49, Part 1 (1989), 143-168.

[15] Ueno, K. and Nakamura, Y., Transformation theory for anti-self-dual equations, Publ. RIMS, 19 (1983), 519-547. 